\author{
Inigo Bocken
}

\title{
Die nomadische Reise der Theorie: Michel de Certeaus Theorie der Alltäglichkeit und die neuzeitliche Mystik
}

Schlüsselwörter: Alltäglichkeit, Gesellschaftskritik, Zentrum und Marge, Nomadische Mystik, Theorie

DOI: 10.4312 /ars.10.2.93-107

Migration ist nicht nur eine soziale Realität. Sie scheint ebenso eine Figur zu sein, die einige Wesenszüge der modernen Kultur überhaupt freilegt und die sogar für ein Wirklichkeitsverhältnis steht, das wir als solches nur mit der Moderne verbinden, oder - um es noch anders zu sagen -, die etwas über die moderne conditio humana aussagt, wie sie seit der frühen Neuzeit auch unsere Zeit noch prägt. Für den Historiker, Psychoanalytiker und Kulturwissenschaftler Michel de Certeau (1925-1986) ist die Bewegung der Migration als unaufhörliche Bewegung von einem Standpunkt zu einem anderen fast symbolhaft für das Verständnis des religiösen Bewusstseins der Moderne, in der die traditionelle Fassung des Christentums ihre Selbstverständlichkeit hat aufgeben müssen. Doch diese unaufhörliche Bewegung des Verlassens des Mittelpunktes und das Sich-Wieder-Auffinden in der Marge ist nicht nur charakteristisch für das Verständnis des Religiösen - viel mehr scheint dieser Verlust der Selbstverständlichkeit paradigmatisch zu sein für die Art und Weise, wie alle gesellschaftlichen Praktiken sich in einer modernen Kultur, die so geprägt ist von der Dominanz einer bestimmten technokratischen Rationalität der Kontrolle, verhalten. Dieser Beitrag möchte aufzeigen, wie der Historiker und Kulturtheoretiker Michel de Certeau Migration als Folge einer technokratischen Rationalitätsfigur betrachtet und dabei nicht nur negativ als Verlust eines Zuhause-Seins versteht, sondern auch Möglichkeiten erkennt, sie als positive Figur einer Gesellschaftskritik zu verstehen. Seine diskursanalytische Betrachtungsweise sieht in der Gestalt des Reisenden und des Nomadentums ein Paradigma für den Widerstand des Menschen im Alltag gegen die Allgegenwärtigkeit der technokratischen und ökonomischen Vernunft. Zugleich versteht Certeau seine Theorie des Reisens als eine Kritik an den dominanten sozialwissenschaftlichen Methoden des ausgehenden 20. Jahrhunderts. Dabei greift er zurück auf die Erfahrung der Mystiker der frühen Neuzeit, die er als Historiker der Spiritualität gründlich erforscht hat. Im ersten Teil dieses Beitrags wird 
Certeaus Theorie der Alläglichkeit (L'invention du quotidien, 1980) vorgestellt, und zwar als eine neue kulturwissenschaftliche Disziplin und als eine Theorie, in der die Bewegung vom einen Standpunkt zum anderen eine Art Gesellschaftskritik, genauer eine Kritik an den herrschenden Sozialwissenschaften, darstellt. Im zweiten Teil wird diese Theorie vor dem Hintergrund der Geschichte der neuzeitlichen Mystik beschrieben, wie sie in La fable mystique (Mystische Fabel, 1984) entfaltet wird, als eine Geschichte der Bewegung zwischen Standpunkten, als ein ständiges Verlassen des Mittelpunktes. Im Schlussteil werden einige weiterführenden Perspektiven skizziert, die die Relevanz dieser Theorie für das Verständnis der Migration in den Kulturwissenschaften erläutern können.

\section{Der Standpunkt der Alltäglichkeit}

Michel de Certeau fand mit seiner Theorie des Alltäglichen bzw. des Alltagslebens anfangs vor allem in den USA großen Anklang und erreichte erst in den letzten Jahren auch in Europa das Zentrum der intellektuellen Debatte. Gerade deshalb soll hier von Michel de Certeau die Rede sein, weil in seinem Werk das Reisen eine so entscheidende Rolle spielt - die Bedeutung der Bewegung vom Mittelpunkt zur Peripherie und umgekehrt. Diese Bedeutung ist, wie hier näher gezeigt wird, so ausschlaggebend, dass sie auch seine Auffassung des Verhältnisses von Theorie und Praxis bestimmt. Genauer formuliert ist die Erfahrung des Standpunktwechsels ausschlaggebend für die Einsicht in die Möglichkeiten und Grenzen der Theorie in Hinsicht auf die Praxis. Wenn Michel de Certeau so interpretiert wird, dass der theoretische Standpunkt mit einem Reiseführer vergleichbar ist, dann wird auch die kritische Bedeutung dieser Auffassung für unsere Zeit deutlich. Leben wir doch in einer Zeit, in der meist unbeholfen nach einem Standpunkt gesucht wird, von dem aus das Ganze der Wirklichkeit bedeutungsvoll wird. Die Unbeholfenheit ist nicht frei von Gefahren, denn sie verleitet zu einem voreiligen Begreifen von Wirklichkeiten, die eigentlich unsere Einsicht übersteigen. Und damit sind nicht nur die großen Ideologien des zwanzigsten Jahrhunderts gemeint, die ihre Unbeholfenheit mit der Ausrottung ganzer Bevölkerungsgruppen kompensierten, sondern auch die schleichende Allmacht der einseitigen und allzu einfach einleuchtenden ökonomischen Modelle oder der allgegenwärtige Hang zur Kontrolle und Erfassung, der durch die digitale Entwicklung noch verstärkt wird - um nur einige Beispiele zu nennen für die Tendenz, der Unbeholfenheit einer Theorie ,von oben herab - ,the view from nowhere' (wie Thomas Nagel es so treffend ausdrückte) - nicht ins Auge sehen zu wollen. ${ }^{1}$ 
Die gefährliche Unbeholfenheit einer Theorie kommt auch weniger dramatisch in der weit verbreiteten Erfahrung zum Ausdruck, dass Organisationen in einer zunehmend komplexer werdenden Wirklichkeit die Komplexität mit zunehmenden Regulierungen bekämpfen, in denen autonome Entscheidungen eine immer weniger relevante Rolle spielen. Gleichzeitig kann die paradoxe Entwicklung beobachtet werden, dass gerade aufgrund derselben Komplexität ein zunehmender Ruf nach Kreativität und Selbstständigkeit im Umgang mit diesen Regeln und Codes zu vernehmen ist. Alles muss geregelt werden - von oben herab -, während zugleich autonome und kreative Positionen notwendig sind. Es scheint unmöglich zu sein, den eigenen Standpunkt einzunehmen und sich zugleich dem System zu unterwerfen. Dieses Paradox, das unsere Kultur kennzeichnet, ist nicht aussichtslos, aber es verlangt nach einer Neupositionierung des theoretischen Bewusstseins, des Verhältnisses zwischen Theorie und Praxis, einer erneuten Reflexion über den Standpunkt von oben herab, über den view from nowhere. Die Frage ist deshalb, wie man sich zwischen diesen Standpunkten bewegen kann, ohne den eigenen Standpunkt loslassen zu müssen genau die Frage also, die mit Migrationsprozessen verbunden ist. Innere Emigration gehört zu den Denkfiguren, die sich in der Neuzeit entwickelt haben, doch ist diese historische Gestalt nur eine Variation der Suche nach Standpunkten, von denen aus man kreativ mit der Wirklichkeit umgehen kann. Im Folgenden soll aufzeigt werden, dass Certeaus Theorie der Alltäglichkeit als eine Suche nach Orten verstanden werden kann, von denen aus ein Individuum sich den herrschenden Diskursen entziehen kann, ohne gleich von der Gesellschaft losgelöst zu sein.

Dieses Problem kann im Rahmen des vorliegenden Aufsatzes nicht ausführlicher behandelt werden. Aber es bildet den Hintergrund für die Weise, in der die TheorieAuffassung von Michel de Certeau behandelt werden soll und die mit dem Begriff eines Reiseführers angedeutet wird. Denn die Bedeutung seiner Theorie des alltäglichen Lebens als theoretische und kulturelle Herausforderung ist bis heute - ungeachtet der intensiven Rezeption in den amerikanischen cultural studies - nicht ausreichend erkannt worden. ${ }^{2}$

Im Folgenden wird zuerst auf Certeaus Alltagstheorie und auf die Weise, in der hier die Perspektive des, gemeinen Menschen ' im Zentrum steht, eingegangen. Vor allem soll gezeigt werden, welchen Platz die Theorie für Certeau einnimmt. Anschließend wird auf Certeaus Studie über die Mystik in der frühen Neuzeit (sechzehntes und siebzehntes Jahrhundert) eingegangen, die den Hintergrund für Certeaus Kulturtheorie bildet. Mit einigen Betrachtungen über den Theoretiker als Reisenden wird der Artikel schließlich abgerundet.

2 Vgl. Ian Buchanan, Michel de Certeau-Cultural Theorist, S. 2. 


\section{L'homme ordinaire - der gemeine Mensch}

In seinem 1980 erschienen Buch L,invention du quotidien 1: Arts de faire (Die Erfindung des Alltäglichen, besser bekannt unter dem Untertitel: Arts de faire/Kunst des Handelns) untersucht Certeau die den alltäglichen Praktiken eigene Kreativität, die sich den herrschenden wissenschaftlichen und kulturellen Diskursen entzieht. Die zentrale Gestalt des Buches ist der ,gemeine Mann' (gemeint sind auch die Frauen), der ,Held des Alltags', dessen Perspektive dem modernen und zeitgenössischen theoretischen Bewusstsein fast völlig äußerlich bleibt. ${ }^{3}$ Certeau zufolge ist gerade der Ausschluss dieser Perspektive kennzeichnend für die moderne Rationalität, die von Beherrschungsstrategien und disziplinierenden Diskursen geprägt ist. Zwar ist das Leben des Alltags schon längst Gegenstand (sozial)wissenschaftlicher Forschung und theoretischer Betrachtung, doch immer wieder - und allmählich öfter - werden die alläglichen Praktiken als anonyme Strukturen, Funktionen und Diskurse beschrieben, die Individuen bestimmen und sogar hervorbringen. Der, gemeine Mann' erscheint in diesen Analysen und Beobachtungen vor allem als das dunkle, unbekannte ,Andere der Diskurse. ${ }^{4}$ Obwohl die neuzeitliche Theorie von sich selber behauptet, sich primär an der Praxis zu orientieren, verhält sie sich dennoch als ein Betrachter von außen, der letztendlich einen panoptischen Überblick über die Bahnen der kulturellen und gesellschaftlichen Praktiken anstrebt und für sich beansprucht. Certeau kritisiert die Grundtendenzen in den Kultur- und Sozialwissenschaften, die die konkreten Praktiken sozusagen ausschließen und eine Betrachtungsweise von außen einnehmen. Auch Michel Foucault, der sich so intensiv der Analyse der Disziplinierungsstrategien und Praktiken gewidmet hat, entkommt dieser Reduktion nicht ganz. ${ }^{5}$ Denn fast alle wissenschaftlichen Analysen der kulturellen Praktiken umgehen die Perspektive des ,gemeinen Menschen', indem sie ihn nur als passiven Teilnehmer beschreiben, der zur Anpassung verurteilt ist. Auch wenn Certeau auf den ersten Blick theoretisch mit Foucault verwandt zu sein scheint, wirft er ihm dennoch vor, sich so sehr auf die Unumgänglichkeit der Machtdispositive konzentriert zu haben, dass er nicht mehr in der Lage gewesen sei, die eigene Kreativität des regen Alltags zu thematisieren - ein Auge zu haben für die Art und Weise, auf die Menschen, die den Diskursen unterworfen sind, mit diesen umgehen, darauf reagieren und sich der panoptischen Kontrolle der technokratischen Modernität entziehen.

Certeau ist nicht so sehr daran interessiert, neue Wege $\mathrm{zu}$ finden, um die soziologische oder kulturelle Figur des gewone man (ordinary man, gemeinen

3 Vgl. Michel de Certeau, L'invention du quotidien. Tome 1: Arts de faire, S. 7 und weiter.

4 Ibid., S. 1.

5 L'invention du quotidien, S. 22. 
Menschen, l'homme ordinaire) zu begreifen. Vielmehr will er eine Theorie entwickeln, die sich auf die Grenzen und Peripherien der herrschenden Diskurse richtet. Dort ist es nämlich, wo sich die interessanten und kreativen Geschehnisse innerhalb einer Kultur ereignen, wie Certeau bereits in einer früheren Schrift gesagt hatte, die 1974 unter dem Titel La culture au pluriel publiziert wurde. ${ }^{6}$

Gewiss: Der gemeine Mensch bleibt still und anonym - Teil der Masse - und in diesem Sinne liegt Certeaus Verständnis dieser Figur ganz auf einer Linie mit den modernen sozialwissenschaftlichen Theorien des zwanzigsten Jahrhunderts. Was diese Theorien Certeau zufolge jedoch nicht sehen, ist die Tatsache, dass ,gemeine Menschen' im alltäglichen Leben kreative Weisen des Handelns und Tuns entwickeln, die sich dem Blick dominanter Diskurse entziehen, auch, wenn sie sich in ihnen abspielen und in ihnen handeln.

Genau an dieser Stelle führt Certeau seinen berühmten consument ein. Der Konsument ist nichts anderes als der jedermann unserer Zeit und es ist unverkennbar, dass ein leicht ironischer Unterton anklingt, wenn Certeau über einen Konsumenten spricht. Ist doch der Konsument in einer kapitalistischen Gesellschaft gerade der passive Verbraucher dessen, was ihm von tonangebender Seite angeboten wird. Nach Certeau ist es jedoch nötig, die unerwartete Kreativität des Konsumenten zu verstehen. Das ist es schließlich, wovon L'invention du quotidien handelt: die niemals endende Kreativität im Handeln des Konsumenten, der seinen eigenen Weg geht, unkontrollierbar für jede Instanz, was auch immer unternommen wird, um eine Kontrolle einzurichten und zu gewährleisten. ${ }^{7}$

Die Konsumenten - das heißt Kunden des Supermarkts, Fernsehzuschauer, Bürger, die spazieren gehen in von Ingenieuren geplanten Parkanlagen. Certeaus Interesse gilt der Art und Weise, in der Konsumenten mit den organisierten Systemen spielen. Sie sind kreativ im Finden von Schleichwegen, Querstraßen, Abkürzungen. Sie bleiben zwar an die vorgegebene Struktur gebunden, sie können nicht ohne weiteres ihren Standpunkt verlassen, aber sie können sehr wohl die Spielräume finden, die ihr Standpunkt anbietet.

Die neue Wissenschaft, die Certeau in seinem Buch anstrebt, will gerade ein gut geübtes Auge entwickeln für diese verborgene Kreativität von unten her, die sich an den Peripherien abspielt - eine Kreativität, die nicht länger die Kreativität des autonomen und beherrschenden modernen Subjekts ist. Es handelt sich um eine Kreativität, die abhängig ist von den bestehenden geplanten Systemen und Diskursen; gleichwohl ist auch sie eine Form der Kreativität. Es ist die unendlich

6 Vgl. Michel de Certeau, La culture au pluriel.

7 Vgl. Marian Füssel, Tote Orte und gelebte Räume. Zur Raumtheorie von Michel de Certeau S J. 
lebhafte Wirklichkeit (Certeau spricht von wimmelnder Wirklichkeit), die sich nicht nur der Kontrolle von sozialen und kulturellen Systemen entzieht, sondern auch den herrschenden sozialen Theorien - sie sind aus der Sicht Certeaus nichts anderes als eine Variante des kontrollierenden technokratischen Systems moderner Wissenschaft und Rationalität. ${ }^{8}$

Insofern der Fokus der von Certeau postulierten Wissenschaft der alltäglichen Praktiken auf dem anonymen Konsumenten liegt, muss sie ein Narrativ entwickeln, in dem zwei entgegengesetzte Richtungen zur Sprache kommen - die jedoch nicht in symmetrischer Weise entgegengesetzt sind. Denn die subversive Kreativität des Konsumenten, mit der er nicht dem von Ingenieuren geplanten Weg folgt und die stabilen Systeme destabilisiert, hängt selbst auch wiederum von diesen Systemen ab. Das ist auch der Grund, weshalb die Kreativität der ordinary people eine andere ist als die Kreativität, die in der Moderne kultiviert wurde. Wichtig für Certeau ist die Tatsache, dass die Konsumenten nicht mehr ohne weiteres Objekte von Machtsystemen sind. Für ihn existiert kein einziges System (oder kein Diskurs), wie mächtig und beherrschend es auch sein mag, das nicht doch Risse und Öffnungen hätte. Es gibt kein System, das in der Lage wäre, das Ganze der lebhaften Wirklichkeit abzudecken, und es liegt an den Öffnungen und Rissen, den blinden Ecken, dass es Konsumenten möglich ist, um auf komplett unerwartete Weise zu reagieren und zu handeln. Die herrschenden Sozialtheorien haben kein Auge für die Tatsache, dass die Konsumenten ihren Weg in einem Supermarkt suchen können und auf eigene Weise mit der von Managern programmierten Ordnung umgehen. Die Autonomie ist nicht absolut und bleibt abhängig von den Strukturen, in denen sich der Konsument bewegt. Aber keine einzige Struktur kann vermeiden, dass der Konsument sich auf seine/ihre Weise zu dieser Struktur verhalten kann. Der Verbraucher kann sogar, wenn auch dieses Verhältnis selber wieder geregelt wird, sich doch auch wiederum auf seine/ihre Weise zu dieser Regelung verhalten. Dieser Spielraum ist irreduzibel gegeben mit jeder Struktur, wie anspruchsvoll sie auch immer sein mag. Es ist nicht ausgeschlossen, dass die Sensibilität Certeaus für dieses Verhältnis mit seiner tiefen Vertrautheit mit religiösen und spirituellen Traditionen zu tun hat. Als Jesuit weiß Certeau sehr gut, dass auch die Gehorsamkeit eines Ordensmitglieds gegenüber dem Provinzial nur auf die Weise dieses Jesuiten erfolgen kann. Sogar wenn man bereit ist, Gehorsamkeit radikal und bedingungslos anzustreben, kann der individuelle Jesuit es nicht vermeiden, dass er selber nicht anders als auf seine eigene Weise gehorsam sein kann, sein eigenes Gehorsam-Sein muss sich finden in und verbinden mit der Weise, in der er selbst existiert und lebt. Er muss die Gehorsamkeit neu erfinden. Es ist keine Struktur denkbar - wie geschlossen auch immer - die nicht diese Grenze hätte.

8 Vgl. Inigo Bocken und Eveline van Buijtenen, Weerbarstige spiritualiteit. Inleiding in het denken van Michel de Certeau, S. 45. 
Der Provinzial kann zwar Gehorsamkeit verlangen, aber er kann nicht außerdem die eigene Weise von Gehorsamkeit bestimmen, denn selbst, wenn er es täte, müsste der gehorsame Jesuit auch damit wieder auf seine Weise umgehen. ${ }^{9}$ Das soll übrigens kein Plädoyer für jesuitische Gehorsamkeit sein - obschon diese Lebensform nicht wenige, auch irdische, Vorteile birgt - es geht hier darum, zu begreifen, an welche Motive Certeau denkt, wenn er kapitelweise die kreativen Spielräume des Konsumenten, des homme ordinaire, verherrlicht - warum er entgegen den Theorien seiner Zeit von der Unableitbarkeit dieser Kreativität, die sich dem Standpunkt, von oben' entzieht, überzeugt ist.

Diese Überzeugung hat weitreichende Folgen für die Weise, in der Certeau über den Ort der Theorie nachdenkt. Gerade weil er die Unableitbarkeit dieser Schlupfwinkel und Schleichwege denken will, kann die Theorie nicht mehr arglos modern ,von oben' die praktische Wirklichkeit betrachten. ${ }^{10}$ Aufgabe der Theorie ist es vielmehr, die verborgene Kreativität aufzudecken und das Auge zu schulen für die Spielräume, in denen mehr Möglichkeiten liegen, als von oben her sichtbar wird.

Certeau versucht mit seiner Theorie der Alltäglichkeit der Perspektive der Konsumenten - der hommes ordinaire - Rechnung zu tragen, wenn es auch nicht beabsichtigt sein kann, die Gesetze und Prozeduren dieser Freiräume zu beschreiben. Im Gegenteil, denn dies würde bedeuten, dass auch diese , marge selbst wieder von der technokratischen, beherrschenden Vernunft erobert werden würde. Diese marginalen Freiräume haben letztendlich etwas Unendliches - es gibt für den Konsumenten eine unendliche Zahl von Möglichkeiten mit den Prozeduren und Gesetze umzugehen. In diesem Sinne ist der homme ordinaire ein Punkt des Widerstandes, an dem die Regeln und Prozeduren, die Pläne und Programme auf Grenzen stoßen - das Auftreten des Konsumenten ist sozusagen eine Unterbrechung (,rupture`) der Systemlogik und der von den Ingenieuren am Reißbrett entworfenen Programme. Der Konsument hat dabei keine andere Wahl als kreativ zu sein, indem er vor dieser Unterbrechung steht - vor der Frage, wie es sich dann zu den bestimmten Diskursregeln verhalten soll. Und gerade das ist es, was die Theorie der Alltäglichkeit zeigen will: Sie beschreibt Diskurse, Programme und Kontrollsysteme, doch zugleich skizziert sie, wie die möglichen Verhaltensweisen der Konsumenten wie ein Kontrapunkt in den Bereich der Systeme eindringen, wie sie Schatten bewirken und Risse, tote Winkel und blind spots. Dennoch ist es nicht so, dass es bei Certeau zuerst diese Freiräume gäbe, die daraufhin von kreativen Konsumenten genützt werden würden - vielmehr ist das

9 Vgl. Bocken, van Buijtenen, S. 86.

10 Vgl. Koenraad Geldof und Rudi Laermans (Hgg.), Sluipwegen van het denken. Over Michel de Certeau, S. 1-5. 
Umgekehrte der Fall. Es gibt Freiräume, weil es Konsumenten gibt, die - oft und sogar meistens ohne revolutionäre Intention - kreativ mit den Regeln umgehen. Für Certeau ist es klar: Je mehr die technokratische, planende Vernunft versucht, die Kluft zwischen Regeln und Anwendung(en) zu schließen, desto grösser werden die Freiräume mit ihren unendlichen Möglichkeiten. Diese These erinnert an den Performance-Philosophen Bazon Brock, der darauf hinweist, dass der Versuch, Probleme zu lösen, die Probleme nur vergrößert; deshalb soll man, Bazon Brock zufolge, vielmehr Arbeit an unlösbaren Problemen leisten. Das ist ein Gedanke, der mit dem von Certeau sehr verwandt ist. ${ }^{11}$

Nun ist die Frage, wie soll diese Wissenschaft, diese Disziplin der Alltäglichkeit, dies leisten? Wie ist sie in der Lage, die herrschenden Diskurse und die unendliche Unterbrechung zusammen zu denken, ohne ein neues geschlossenes System zu denken? Und wo genau ist der Ort der Theorie in diesem Wechselspiel zwischen technokratischem System bzw. Regelsystem und kreativem Konsumenten? Und ferner: Wie kann Certeau behaupten, dass der Konsument überhaupt kreativ sei? Ist es nicht unsere Erfahrung, dass die meisten Konsumenten einfach machen, was die Mode vorschreibt? Und hat Foucault nicht Recht, wenn er das freie Subjekt für tot erklärt hat, indem wir nur noch Objekte innerhalb von überkomplexen Systemen sind, nur noch ab und zu ausgerüstet mit der Illusion, dass wir etwas Eigenes haben und denken?

Zuerst zur Frage, wie Certeau zufolge die Theorie der Alltäglichkeit in der Lage ist, die creative rupture, die Unterbrechung, zu beschreiben. Es ist klar, dass Certeau sich der Unmöglichkeit bewusst ist, eine Theorie $\mathrm{zu}$ entwerfen, die oberhalb aller Diskurse steht, und so den Widerstand des Konsumenten zu beschreiben. Und gerade hier tritt wieder die Metapher des Reisens, der Bewegung von einem Standpunkt zu einem anderen, in den Vordergrund. Der Theoretiker ist ein Reisender. So hat Certeau auch sich und seine Arbeit in seinem L'Étranger ou l'union dans la différence gekennzeichnet. ${ }^{12}$ Und als Reisender scheint der Theoretiker Certeau sich auch in seinem L'invention du quotidien zu verhalten. Dort ist er aber nicht bloß Reisender - mehr noch ist er ein Reiseführer. Er zeigt dem Leser ein Gebiet, in dem er selbst nicht zuhause ist, das ihm aber trotzdem vertraut ist. Der Reiseführer zeigt seinen Mitreisenden einen bestimmten Weg (une trajectoire), der ganz prägnant die inneren Zusammenhänge der Viertel und Straßen erkennen lässt, manchmal besteigt er einen Aussichtsturm und dann führt er ihn in ein typisches Viertel, in dem noch alte Traditionen zuhause sind. Der Reiseführer ist selbst Reisender, er steht nicht außerhalb des Geschehens, sondern er geht selbst den Weg, kennt sich aus, aber kennt nicht alle

11 Vgl. Bazon Brock, Ein optimistischer Blick auf den Pessimismus, S. 20 und passim.

12 Vgl. Michel de Certeau, Létranger ou l’union dans la différence, S. 21. 
Tiefen und unbekannten Ecken. Er weiß wohl, dass es sie gibt und manchmal kann er seinen Mitreisenden doch einen interessanten Einblick gewähren. Der Reiseführer hat teil am Geschehen, doch anders als die Einwohner: er bleibt ein Fremder in dem Gebiet, jedoch ein Fremder, der sich auskennt (der manchmal einen Bekannten trifft, wie das in La fable mystique der Fall ist, und der manchmal auch wiedererkannt wird). Reiseführer, das heißt eine Position zwischen der Perspektive von oben den strategischen, kontrollierenden Instanzen - und der des homme ordinaire, des Konsumenten, eine Position der taktischen Bewegungen, der Schleichwege und der von oben unsichtbaren Ecken.

In Linvention $d u$ quotidien zeigt Michel de Certeau sich voll und ganz als Reiseführer. Vor allem ist das der Fall im berühmten und oft zitierten Kapitel über Certeaus Spaziergang durch New York. ${ }^{13}$ Die Tatsache, dass dieser Spaziergang vor einem symbolträchtigen Standort anfing, hatte sogar der sensible Certeau im Jahre 1980 nicht ahnen können: Es handelt sich um das 110. Stockwerk des World Trade Center in New York, für Certeau das Symbol schlechthin für den Standort der neuzeitlichen Rationalität. Nirgendwo anders in der Welt wird die panoptische Perspektive so explizit verwirklicht wie hier. Denn von hier aus sieht Certeau das geometrische Muster des Stadtplans, wie es von modernen Stadtplanern und Ingenieuren auf einem Schreibtisch geplant worden ist - genau wie das Paris von Hausmann, der die kurvigen und organisch gewachsen Gassen des mittelalterlichen Paris den geraden und geometrisch geordneten modernen Boulevards der Stadt geopfert hat. Diese geometrischen Strukturen organisieren den Raum, zeigen Verbindungen und bestimmen Funktionen ganzer Stadtteile, deren Bewohner letztendlich miteingeplante Objekte sind.

Es gibt jedoch auch eine andere Perspektive als die des 110. Stockwerks des WTC. Denn Certeau beschreibt in einer fast lyrischen Atmosphäre, wie er mit dem Aufzug hinabfährt und seinen Spaziergang durch die Straßen fortsetzt. Er zeigt dem Leser die unendliche Vielfalt des alltäglichen Lebens in den Straßen, eine Vielfalt, die sich dem Blick von oben entzieht. Der Leser sieht, Certeau folgend, wie die Bewohner ihre eigenen unerwarteten Querwege gestalten, wie neue, nicht aus der Vogelperspektive geplante Praktiken und Gewohnheiten entstanden sind, Bezüge, die nur im repetitiven Tun gewachsen sind. Certeau beschreibt die Spaziergänge der Bewohner, die Abkürzungen nehmen, oder Ampeln meiden. Innerhalb des von oben geplanten Rahmens schaffen die Bewohner ihre eigenen Wege und Lebensmuster, sie sind in der Lage tote Winkel und blind spots zu benützen und gestalten so eine Stadt, die anders ist als die von den Ingenieure programmierte. 
So, d.h. als Reiseführer, der sich durch die Stadt bewegt und Grenzen überschreitet, ist der Theoretiker in der Lage, die beiden Perspektive zu zeigen, er weist auf die Orte hin, an denen sie sich treffen oder gerade auseinandergehen, er zeigt, was von einer Seite unsichtbar ist.

Es sind gerade diese Orte, die Risse innerhalb von geschlossenen Systemen aufzeigen, die eine unendlich Zahl von Möglichkeiten von Verhaltensweisen aufzeigen, mit denen der Konsument sich innerhalb der geometrischen Figuren bewegen kann und wo er mit seiner partiellen Unsichtbarkeit spielen kann.

Der reisende, Grenzen überschreitende Theoretiker weiß um den Standpunkt von oben - den view from nowhere - doch er befindet sich nicht dort - er reist durch die Räume, in denen die hommes ordinaires sich auf immer unerwartete Weise zu den Strukturen verhalten, in denen sie leben. So lernt und lehrt der Theoretiker zu sehen, was fremd für ihn ist, er entdeckt andere und unerwartete Perspektiven, die er niemals selbst einnehmen kann und die sich teilweise auch seinem Blick entziehen. Letztendlich muss der Leser die Reise selbst machen - auch das gehört zum Bild des Theoretikers als Reiseführer.

\section{Mystik}

Es bleibt jetzt noch die Frage nach der widerspenstigen Kreativität des Konsumenten. Woher nimmt Certeau diesen Gedanken und warum unterscheidet er sich so ausdrücklich von seinem Landsmann Michel Foucault und seiner Analyse der Macht? Wieso ist er sich so sicher, dass es immer diese Kreativität des homme ordinaire ist? Ein Schlüssel zur Beantwortung dieser Fragen liegt in der Beschäftigung Certeaus mit der Geschichte der frühneuzeitlichen Mystik. Bekanntlich hat Certeau sich oft - und bis zu seinem Lebensende - als Reisender in dem vielfältigen Land der Mystik des 16. und 17. Jahrhunderts aufgehalten. Dabei soll nicht vergessen werden, dass Mystik - als Substantiv verstanden - für Certeau ein typisch neuzeitliches Phänomen ist. Die Mystiker sind gerade diejenigen, die über den Verlust trauern, über die Abwesenheit Gottes, von der die neuzeitliche Kultur so geprägt ist. ${ }^{14}$

So bekommt er ein Auge für das, was ihm fremd ist, er entdeckt, dass es andere Perspektiven und Standpunkte gibt, die er selbst niemals einnehmen kann und die sich teilweise seinem Blickfeld entziehen. Aber er kann seinen Blick für die vielen Weisen üben, in denen Menschen mit den dominanten Diskursen umgehen, die nach Peripherien und toten Ecken suchen oder das manchmal gerade nicht tun.

14 Vgl. Michel de Certeau, La fable mystique. XVIème et XVIIème siècle, Tome 1, S. 12-20, passim. 
Die Trauer und die Melancholie der Mystiker bilden eine neue Disziplin, ein neues Sprechen am Rande der herrschenden gesellschaftlichen Diskurse. Aufgrund der Trauer und der Melancholie, die von den Mystikern in ihrer paradoxen Sprache kultiviert wird, bewegen sie sich auf der Grenze der technokratischen Diskurse, die, wie Certeau es formuliert, von der écriture, der geschriebenen Sprache, also der kontrollierenden technokratischen Vernunft gekennzeichnet sind. Von Teresa van Avila bis Jean de Saint-Samson, von Jean-Joseph Surin bis Jean De Labadie, sagen alle aus, dass Gott in seiner Schöpfung schweigt, und bringen die écriture mittels Paradoxa und Oxymora bis an die Grenze ihrer Vermögen - so machen Trauer und Leere in der Mitte der neuzeitlichen Diskurse eine Erinnerung präsent - und gerade das ist es, was Mystiker tun - mittels Sprache oder im Handeln die Risse und Öffnungen, die Schatten und toten Winkel präsent zu machen.

Es gibt in diesem Rahmen eine besondere Figur, die von Certeau angeführt wird. Am intensivsten tritt sie in der Beschreibung von Jean-Joseph Surin hervor, einem gelehrten Jesuiten und Theologen, dem in der Kutsche nach Rouen ein junger ungebildeter Hirte begegnet, der ihm die tiefsten Geheimnisse offenbart. Was diese Geheimnisse genau beinhalten, wird im Schreiben von Surin nicht erläutert, wichtiger ist es jedoch, dass es eine solche Figur gibt. ${ }^{15}$ Der Brief von Surin, in dem diese Begegnung beschrieben wird, erreicht ein sehr großes Publikum, er wird kopiert und variiert. Die Figur scheint an etwas zu appellieren, was vielen bekannt ist. Certeau zeigt sehr schön, wie dieser einfache Hirtenknabe in der Tradition des idiota steht, den man bei Cusanus und - Certeau zufolge - auch bei Tauler findet, in einer Tradition, die Certeau weiter verfolgt bis zu den Kannibalen von Montaigne. Die Tradition geht sogar noch weiter zurück, bis zu Jan van Ruusbroec, dem großen flämischen Mystiker aus der Tradition der devotio moderna - denn der von Ruusbroec beschriebene ghemeyne mensch weist auf die höchste Phase des mystischen Lebens hin. Es geht dort um die Vereinigung von contemplatio und actio, um den Moment, in dem der mystische Mensch nicht mehr nur die Einigung mit Gott genießt, sondern in die Welt zurückkehrt und im alläglichen Leben handelt. Diese Tradition steht also im Hintergrund von L'invention $d u$ quotidien und seines, homme ordinaire‘, der an einer anderen Stelle im Text auch homme commun, mittelniederländisch also ghemeyne mensch, genannt wird.

Diese Gestalt - der idiota, ghemeyne mensch (Ruusbroec), Hirtenknabe, Kannibale - scheint ein ständiger Wegbegleiter des Theoretikers Michel de Certeau zu sein, der durch das große Land der neuzeitlichen Mystik reist. Auf dieser Reise folgt er JeanJoseph Surin auf dem Weg nach Rouen, er folgt Jean de Labadie auf dem Fuß, also Labadies Reise von Ort zu Ort, wenn er vom Jesuiten zum Priester, vom Priester zum Jansenisten, vom Jansenisten zum Calvinisten, und endlich vom Calvinisten zum

15 Vgl. La fable mystique, Tome I, S. 280-306. 
Labadisten wird und dergestalt von einigen treuen Anhängern in einem Garten in Altona begraben wird. ${ }^{16}$ Und immer wieder betont Certeau, dass er ihn verfolgt, ihn letztendlich doch gehen lassen muss. Nur noch die Spuren im Sand sind da, wie einst bei Empedokles. Er verfolgt Labadies Weg, doch kann er am Ende nicht mit einem solchen Tempo mithalten, er lässt ihn gehen. ${ }^{17}$

Anders ist es mit Cusanus. Der Text über Cusanus ist der wichtigste Text des zweiten Bandes von La fable mystique, der erst im Jahre 2013, 27 Jahre nach Certeaus frühem Tod, erschienen ist. Teile des Cusanus-Textes waren schon in verschiedenen Sprachen erschienen, doch gerade die Teile, die das Reisen des Cusanus betreffen, sind erst jüngst zugänglich geworden. Denn Certeau setzt in diesem Text alle ihm verfügbaren theoretischen Instrumente ein, um die rastlosen Reisen des Cusanus zu verfolgen. Nur ab und zu verliert Certeau ihn aus dem Auge, z.B. wenn der Kardinal und hilflose Bischof Cusanus sich, um sein Leben fürchtend, in den Alpen versteckt. Ganz nah ist Certeau Cusanus jedoch, wenn er die Inszenierung von De visione Dei beschreibt und deren Dynamik von innen heraus nachzuvollziehen versucht. Denn das Wechselspiel der Perspektiven, das Certeau bei Cusanus findet, die ständigen Unterbrechungen des Sehens, die unerwarteten Perspektivwechsel - das alles entspricht voll und ganz der Art und Weise, wie die Perspektive von oben und die von unten in Linvention $d u$ quotidien miteinander ringen.

\section{Schlussbetrachtung}

So betrachtet hat die Theorie, Certeau zufolge, kein festen Ort. Sie reist herum, schaut herum, schaut nach oben oder nach unten. Sie hilft dem Reisenden besser zu sehen, erblickt unerwartete Perspektiven und sie muss ständig ihre Position suchen. Gerade deshalb ist sie eine Theorie in der Mitte des alltäglichen Lebens, eine Theorie die im Vertrauten das Fremde sucht und im Fremden das Vertraute.

So ist Theorie, nach Michel de Certeau, in der Moderne selbst ständig auf der Suche nach einem Ort, überschreitet Grenzen. Sie hat seit dem späten Mittelalter ihren festen Ort verloren und diese historische Erfahrung ist es, die die Theorie in einer Zeit prägt, in der Grenzen ständig hinterfragt werden. Diese sind manchmal durchlässig, manchmal auch artifiziell gesetzt und fortifiziert, genau wie auch in der konkreten Gesellschaft. Die Kulturtheorie von Michel de Certeau eröffnet den Blick für solche Erfahrungen und versucht sie als ursprüngliche Erfahrungen unserer Zeit vor dem Hintergrund der Geschichte zu deuten.

16 La fable mystique, Tome I, S. 384 und weiter.

17 Vgl. Graham Ward, Michel de Certeau’s Spiritual Spaces. 


\section{Literatur}

Bocken, I., van Buijtenen, E., Weerbarstige spiritualiteit. Inleiding in het denken van Michel de Certeau, Heeswijk 2016.

Brock, B., Ein optimistischer Blick auf den Pessimismus, Klagenfurt 2012.

Buchanan, I., Michel de Certeau - Cultural Theorist, London 2000.

Certeau, M. de, L'invention du quotidien. Tome 1: Arts de faire, Paris 1980.

Certeau, M. de, La culture au pluriel, Paris 1980.

Certeau, M. de, La fable mystique. XVIème et XVIIème siècle, Paris 1984.

Certeau, M. de, Létranger ou l'union dans la différence, Paris 1991 (erste Edition 1969).

Füssel, M., Tote Orte und gelebte Räume. Zur Raumtheorie von Michel de Certeau S J, in: Historical Social Research 38, 2013, S. 22-39.

Geldof, K., Laermans, R. (Hgg.), Sluipwegen van het denken. Over Michel de Certeau, Nijmegen 1996, S. 1-5.

Nagel, T., The View from Nowhere, Oxford 1989.

Ward, G., Michel de Certeau's Spiritual Spaces. South-Atlantic Quarterly 100/2, 2001, S. 501-517. 


\section{Inigo Bocken}

\section{Nomadsko popotovanje teorije: teorija vsakdanjosti Michela de Certeauja in mistika zgodnjega novega veka}

Ključne besede: vsakdanjost, družbena kritika, središče in rob, nomadska mistika, teorija

Izkušnja migracije je za Michela de Certeauja (1925-1986) temelj njegove teorije vsakdanjosti, ki velja za kritiko novoveške tehnokratske racionalnosti in dominantnih socioloških družbenih modelov ob koncu 20. stoletja in ki je bila tudi mišljena kot taka. Michel de Certeau v zgodovinskih študijah o mistiki zgodnjega novega veka opisuje, kako so mistiki kot moderni nomadi popotovali po Evropi, pri tem pa so popotovanje razumeli kot način, da se izmaknejo vladajočim diskurzom moči. Popotovanje mistikov preko meja je tako rekoč paradigmatično za teorijo družbe s perspektive družbenega obrobja in odpira prostor »drugemu«. Poglobljeno študijo o mistikih iz 16. in 17. stoletja lahko razumemo kot ozadje, pred katerim Certeau razvija teorijo vsakdanjosti - pri tem gre za knjigo L'invention du quotidien, ki je močno zaznamovala zlasti novejše kulturološke študije v ZDA. Prispevek razvija in utemeljuje tezo, da ta teorija popotovanje mistikov predpostavlja kot paradigmo. »Navadni« človek je izhodišče tega novega teoretskega modela. Iz tega sledi, da se mora vsakršno kulturološko raziskovanje soočiti z dejstvom, da teorija nima določenega in trdnega mesta. Teorija sama dandanes postaja nomadska. Tako se odpirajo možnosti, da izkušnje migracij danes razvijamo naprej. 


\section{Inigo Bocken}

\section{Nomadic Theory - Michel de Certeau's Theory of Everyday Life and Early Modern Mysticism}

Keywords: everyday life, social criticism, centre and margin, nomadic mysticism, theory

The experience of migration is in the view of Michel de Certeau (1925-1986) the basis of his theory of everyday life. This theory can be understood as the foundation of a critique on modern 'technical' rational, which is so dominant since Early Modernity. This experience is meant to be the foundation of a critique on all forms of social sciences at the end of the $20^{\text {th }}$ Century - until today. In his historical studies on mysticism in Early Modernity Michel de Certeau describes the figure of the mystique as a modern nomad, travelling throughout Europe. This nomadic travelling however is at the same time a metaphorical figure for the mystique's way of escaping and criticizing the dominant discourses of technocratic power. The travelling of the mystique offers so to say a paradigm for a social theory from the perspective of society's margins, opening space for the 'other' (in various meanings). As such Certeau's extensive studies on mysticism in the $16^{\text {th }}$ and $17^{\text {th }}$ Century can be understood as the horizon for his theory of everyday life in his book L'invention du quotidien (translated in English as Everyday Life Practices). This book was quite influential in the United States mainly for the newer cultural studies. In this contribution I argue for the thesis that the travelling of the early modern mystique is presupposed in the theory of everyday life as a kind of paradigm. The 'common man' is, in this book, the starting point for a new theoretical model for cultural studies. It is a perspective showing that every theory in cultural studies has to deal with the undetermined place of theory. Theory itself became, in the view of Certeau, nomadic. From this point of view the experience of migration in our days can be interpreted in innovative ways. 